\title{
Advances in organic 2D crystals
}

\author{
Xinliang Feng \\ Center for Advancing Electronics Dresden \& Faculty of Chemistry and Food Chemistry, Technische Universitaet Dresden, \\ Mommsenstr. 4, 01069 Dresden, Germany; Max Planck Institute of Microstructure Physics, Germany)
}

xinliang.feng@tu-dresden.de

Over the last decade, the discovery of graphene has triggered a new paradigm of two-dimensional (2D) crystal materials. They are characterized by a periodic network structure and topographical thickness at the atomic/molecular level, enabling the investigation of fundamental exotic physical and chemical properties down to a single-layer nanosheet. Thereby, robust technologies and industrial applications, ranging from electronics and optoelectronics to energy storage, energy conversion, membranes, sensors, and biomedicine, have been inspired by the discovery and exploration of such new materials.

In contrast to the tremendous efforts dedicated to the exploration of graphene and inorganic 2D crystals such as metal dichalcogenides, boron nitride, black phosphorus, metal oxides, and nitrides, there has been much less development in organic 2D crystalline materials, including the bottom-up organic/polymer synthesis of graphene nanoribbons, 2D metal-organic frameworks, 2D polymers/supramolecular polymers, as well as the supramolecular approach to $2 \mathrm{D}$ organic nanostructures. One of the central chemical challenges is to realize a controlled polymerization in two distinct dimensions under thermodynamic/kinetic control in solution and at the surface/interface. In this talk, we will present our recent efforts in bottom-up synthetic approaches towards novel organic 2D crystals with structural control at the atomic/molecular level and beyond. We will introduce a surfactant-monolayer assisted interfacial synthesis (SMAIS) method that is highly efficient to promote supramolecular assembly of precursor monomers on the water surface and subsequent 2D polymerization in a controlled manner. 2D conjugated polymers and coordination polymers belong to such materials classes. The unique structures with possible tailoring of conjugated building blocks and conjugation lengths, tunable pore sizes and thicknesses, as well as impressive electronic structures, make them highly promising for a range of applications in electronics and spintronics. Other application potential of organic 2D crystals, such as in membranes, will also be discussed.

Chem. Commun. 2009, 6919; Adv. Mater. 2015, 27, 403; Angew. Chem. Int. Ed. 2015, 54, 12058; J. Am. Chem. Soc. 2015, 137, 14525; Nature Comm. 2015, 6, 8817; Nature Comm. 2016, 7, 13461; Polym. Chem. 2016, 7, 4176; Angew. Chem. Int. Ed. 2017, 56, 3920; Chem. Rev. 2018, 118, 6189; Nature Comm. 2018, 9, 1140; Nature Comm. 2018, 9, 2637; Nature Mater. 2018, 17, 1027; Angew. Chem. Int. Ed. 2018, 130, 13942; Nature Comm. 2019, 10, 3260; Nature Chem. 2019, 11, 994; Nature Comm. 2019, 10, 4225; Nature Comm. 2020, 11, 1409; J. Am. Chem. Soc. 2020, 142, 12903; Angew. Chem. Int. Ed. 2020, 59, 8218; Angew. Chem. Int. Ed. 2020, 59, 6028; Angew. Chem. Int. Ed. 2020, 59, 23620; Science Adv. 2020, 6, eabb5976; Nature Mater. 2021, 20, 122; Chem. Soc. Rev. 2021, 50, 2764; Angew. Chem. Int. Ed. 2021, 60, 13859.

Keywords: graphene; 2D crystals; organic crystalline materials; SMAIS method; controlled polymerization; 2D conjugated polymers 